\title{
A corresponding Cullen-regularity for split-quaternionic-valued functions
}

\section{Ji Eun Kim*}

\section{"Correspondence:}

jeunkim@pusan.ac.kr

Department of Mathematics,

Dongguk University, Gyeongju-si,

38066 , Republic of Korea

\begin{abstract}
We give a representation of the class of Cullen-regular functions in split-quaternions. We consider each Cullen's form of split-quaternions, which provides corresponding Cauchy-Riemann equations for split-quaternionic variables. Using Cullen's form, we research hyperholomorphy and the properties of functions of split-quaternionic variables which are expressed by hyperbolic coordinates.
\end{abstract}

MSC: Primary 32A99; secondary 32W50; 30G35; 11 E88

Keywords: split-quaternion; Cullen's form; regular function; Cauchy-Riemann system; Clifford analysis

\section{Introduction}

The skew field of real quaternions, denoted by $\mathbb{H}$, has the form

$$
\mathbb{H}=\left\{q \mid q=x_{0}+i x_{1}+j x_{2}+k x_{3}, x_{r} \in \mathbb{R}(r=0,1,2,3)\right\},
$$

where $\mathbb{R}$ is the set of real numbers and $i, j$, and $k$ are imaginary units with

$$
\begin{aligned}
& i^{2}=j^{2}=k^{2}=-1, \\
& i j=-j i=k, \quad j k=-k j=i, \quad k i=-i k=j .
\end{aligned}
$$

Theories and applications of functions of a quaternionic variable have been led by holomorphic functions of one complex variable. Quaternions were introduced by Hamilton [1] in 1843 and generalized by Clifford [2] in 1873. After then, Fueter [3, 4] defined differential operators, called the Cauchy-Fueter operators, and regular functions in the space of solutions of the equation with these operators. Kim et al. [5, 6] obtained the regularity of functions on the form of reduced quaternions in Clifford analysis.

Split-quaternions are elements of four-dimensional algebra introduced by Cockle [7] in 1849. Similar to quaternions, they form a four-dimensional real vector space which is a general associative, but non-commutative, form of multiplication. Unlike the quaternions, the split-quaternions contain zero divisors, nilpotent elements, and nontrivial idempotents such as $\frac{(1+j)}{2}$ is an idempotent zero-divisor, and $i-j$ is nilpotent. Indeed, Helmut and Kist [8] constructed an algebra over the real numbers, which is isomorphic to the algebra of $2 \times 2$ real matrices. Moreover, the modulus which has an isotropic quadratic form for

(c) The Author(s) 2017. This article is distributed under the terms of the Creative Commons Attribution 4.0 International License (http://creativecommons.org/licenses/by/4.0/), which permits unrestricted use, distribution, and reproduction in any medium, provided you give appropriate credit to the original author(s) and the source, provide a link to the Creative Commons license, and indicate if changes were made. 
split-quaternions provides hyperbolic motions of the Poincaré disk model of hyperbolic geometry. From this result, the structure of split-quaternion analysis has been developed and applied in four-dimensional physics by Frenkel and Libine [9, 10]. Kim and Shon [11, 12] researched corresponding Cauchy-Riemann systems and the properties of hyperholomorphic functions with values in modified split-quaternions. Kim and Shon [13] gave relations between the properties of split-quaternionic regular functions and their algebraic inverse mapping.

In 1965, Cullen [14] proposed the notion of intrinsic functions and found quaternions convenient to write in the form $\zeta=x_{0}+p \mu$, where $x_{0}$ and $p=\sqrt{x_{1}^{2}+x_{2}^{2}+x_{3}^{2}}$ are real and $\mu$ is a unit vector quaternion. For fixed $\mu$, the elements of the form $x_{0}+p \mu$ constitute a subspace of $\mathcal{D}$ which is isomorphic to the complex field. Since $\mathcal{D}$ is a generalization of the algebra of complex numbers, $\mathcal{D}$ has properties analogous to the class of analytic functions of a complex variable. From these properties, Cullen-regular functions are tried to be related to a class of functions of the reduced quaternionic variable, studied by Leutwiler in [15]. Gentili and Struppa [16] and Alayón-Solarz [17, 18] gave definitions of regularity for functions of a quaternionic variable and developed representations of the Cullen-regularity of quaternion analysis. By using the analytic properties and calculating processes of Cullenregularity, Marin has researched thermoelastic materials in various points of view. For example, Marin and coauthors studied the asymptotic partition of total energy for the solutions of the mixed initial boundary value problem within the thermoelasticity of initially stressed bodies. They obtained a spatial decay estimate which is considered a right cylinder composed of physically micropolar thermoelastic material for which one plane end is subjected to an excitation harmonic in time. Also, they extended the concept of domain of influence in order to cover the elasticity of microstretch materials and studied it for the displacement field, the microrotation field, and the microstretch function (see [19-21]).

Based on these studies, we consider a general type of Cullen-regularity for functions of split-quaternionic variables. First, we give the notions and some properties of splitquaternion-valued functions by using Cullen's form. Also, we investigate the structure of a Cullen-regular function and corresponding split-Cauchy-Riemann systems for Cullenvariables and research properties of hyperholomorphic functions, represented by Cullenregular functions.

\section{Preliminaries}

Let $\mathbb{S}$ denote the skew field of real split-quaternions which has elements of the form

$$
p=x_{0}+i x_{1}+j x_{2}+k x_{3}
$$

where $x_{r}(r=0,1,2,3)$ are real, $i, j$ and $k$ are imaginary units such that

$$
\begin{aligned}
& i^{2}=-1, \quad j^{2}=k^{2}=1, \\
& i j=-j i=k, \quad k j=-j k=i, \quad k i=-i k=j,
\end{aligned}
$$

which is isomorphic to $\mathbb{R}^{4}$. By the properties of the imaginary units of split-quaternions, we have the following rules for addition and multiplication:

$$
p+q=\left(x_{0}+y_{0}\right)+i\left(x_{1}+y_{1}\right)+j\left(x_{2}+y_{2}\right)+k\left(x_{3}+y_{3}\right)
$$


and

$$
\begin{aligned}
p q= & \left(x_{0} y_{0}-x_{1} y_{1}+x_{2} y_{2}+x_{3} y_{3}\right)+i\left(x_{1} y_{0}+x_{0} y_{1}+x_{3} y_{2}-x_{2} y_{3}\right) \\
& +j\left(x_{2} y_{0}+x_{3} y_{1}+x_{0} y_{2}-x_{1} y_{3}\right)+k\left(x_{3} y_{0}-x_{2} y_{1}+x_{1} y_{2}+x_{0} y_{3}\right),
\end{aligned}
$$

respectively. We give the conjugation of a split-quaternion as follows:

$$
p^{*}=x_{0}-i x_{1}-j x_{2}-k x_{3}
$$

Then we have a modulus, denoted by $\mathcal{N}(p)$, and an inverse element, denoted by $p^{-1}$ of $p \in \mathbb{S}$

$$
\mathcal{N}(p):=p p^{*}=x_{0}^{2}+x_{1}^{2}-x_{2}^{2}-x_{3}^{2}
$$

and

$$
p^{-1}=\frac{p^{*}}{\mathcal{N}(p)} \quad\left(x_{0}^{2}+x_{1}^{2} \neq x_{2}^{2}+x_{3}^{2}\right)
$$

respectively. By the non-commutative property of basis vectors $i, j$ and $k$, the product of a split-quaternion with its conjugate is given in an isotropic quadratic form. Given two split-quaternions $p$ and $q$, the following holds:

$$
N(p q)=N(p) N(q)
$$

For any $p \neq 0$ with $N(p)=0, p$ is a null vector, and when the modulus is non-zero, then $p$ has a multiplicative inverse.

For a split-quaternion $p$, it can be written as $p=S(p)+V(p)$, where $S(p)$ is the scalar part and $V(p)$ is the vector part of $p$. Specially, if $S(p)=0$, then $p$ is called pure split-quaternion. We focus the attention on the pure split-quaternions to configure Cullen's form in $\mathbb{S}$. For a pure split-quaternion $p$, we consider $\mathcal{T}=\left\{p=i x+j y+k z \mid-x^{2}+y^{2}+z^{2}=1\right\}$. Since points $(x, y, z)$, which satisfy $-x^{2}+y^{2}+z^{2}=1$, compose Figure 1 in practice, Figure 1 shows that $\mathcal{T}$
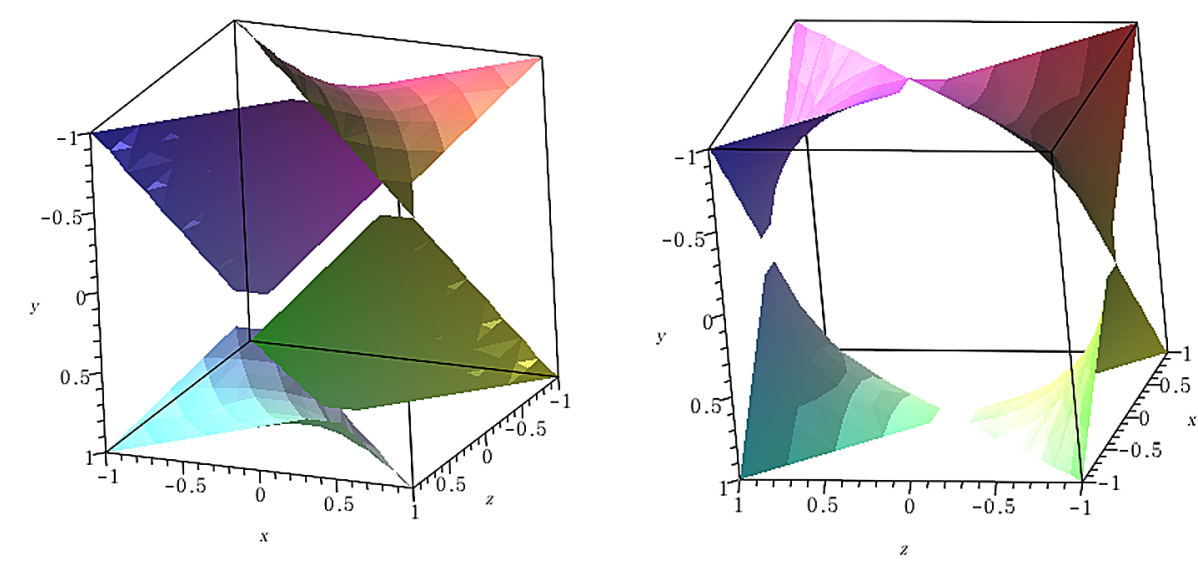

Figure 1 Figures of $-x^{2}+y^{2}+z^{2}=1$ 


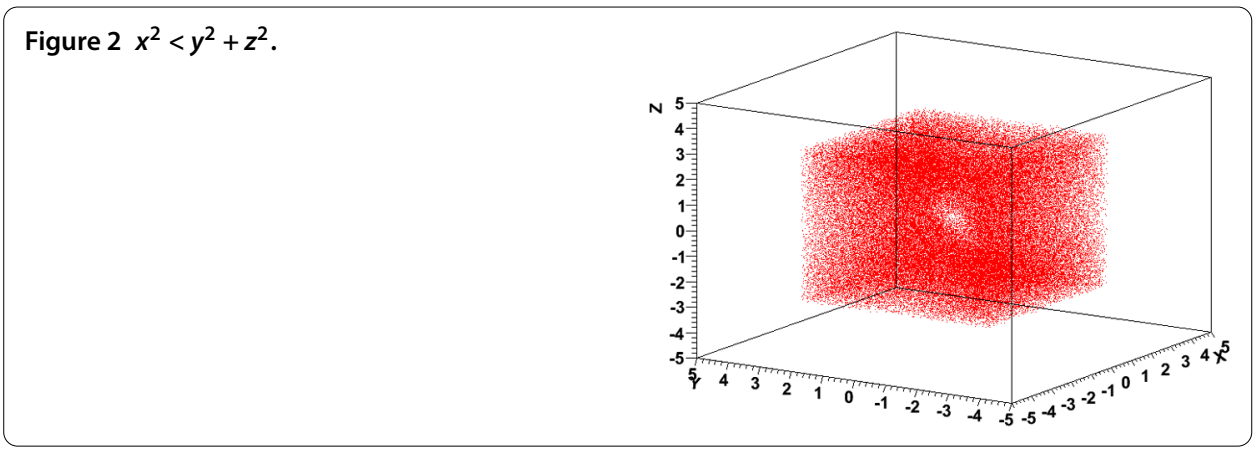

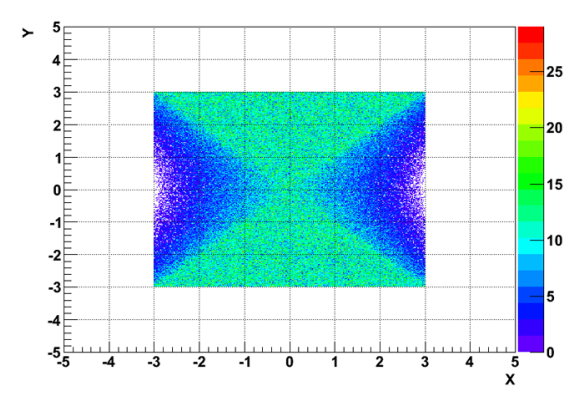

(a) Onto the $x y$-plane

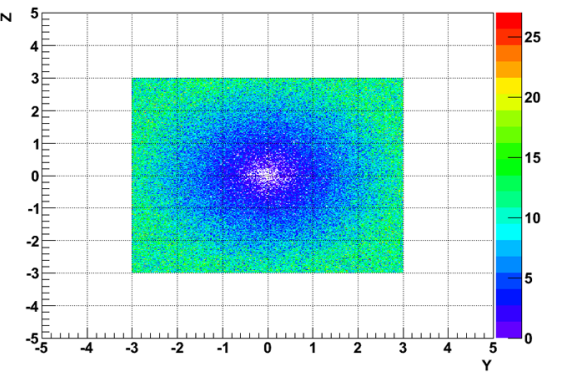

(b) Onto the $y z$-plane

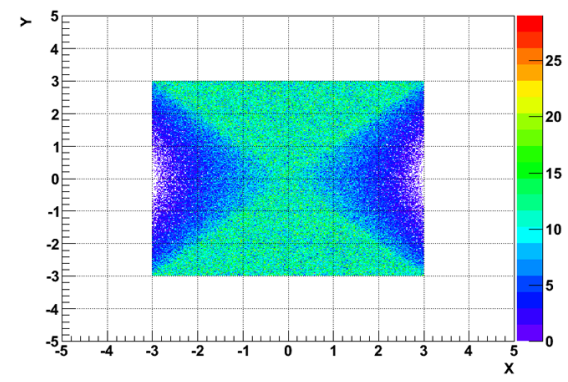

(c) Onto the $x z$-plane

Figure 3 The projection of Figure 2.

can exist and be non-empty in $\mathbb{S}$. So, we can represent the following processes and results. If we let

$$
J=\frac{i x_{1}+j x_{2}+k x_{3}}{\sqrt{-x_{1}^{2}+x_{2}^{2}+x_{3}^{2}}} \quad\left(x_{1}^{2}<x_{2}^{2}+x_{3}^{2}\right),
$$

then $J^{2}=1$ and $J \in \mathcal{T}$. Indeed, the existence of the element $J$ of $\mathcal{T}$ is guaranteed by Figures $2-4$.

Let $\Omega$ be an open set in $\mathbb{S}$. A function $f: \Omega \rightarrow \mathbb{S}$ is given by

$$
f(p)=f_{0}+i f_{1}+j f_{2}+k f_{3},
$$

where $f_{r}=f_{r}\left(x_{0}, x_{1}, x_{2}, x_{3}\right)(r=0,1,2,3)$ are real-valued functions. We try to express a function using the element $J$ of $\mathcal{T}$. So, we consider the class of split-quaternionic functions 


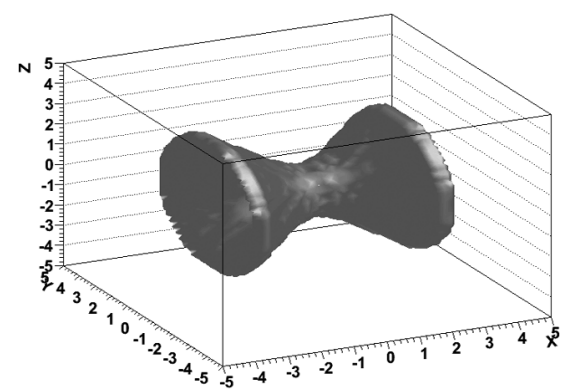

(a) Figure of $\mathcal{T}$

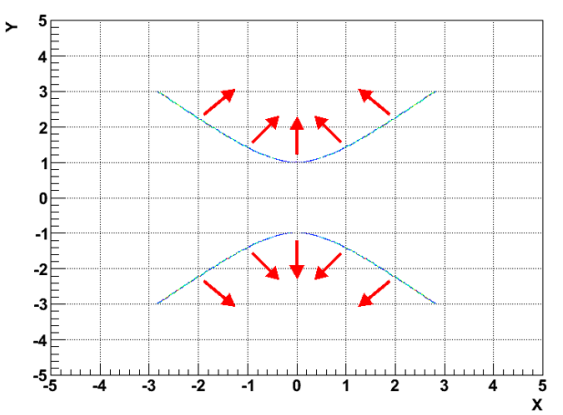

(b) Figure of $n(p)$ on the $x y$-plane of $\mathcal{T}$

Figure 4 Figure of $n(p)$ on $\mathcal{T}$.

which has an element $f$ written as

$$
f=u+J v
$$

by letting $u=f_{0}$ be a real-valued function and

$$
\begin{aligned}
v= & \frac{1}{\sqrt{-x_{1}^{2}+x_{2}^{2}+x_{3}^{2}}}\left\{\left(-x_{1} f_{1}+x_{2} f_{2}+x_{3} f_{3}\right)+i\left(-x_{2} f_{3}+x_{3} f_{2}\right)\right. \\
& \left.+j\left(-x_{1} f_{3}+x_{3} f_{1}\right)+k\left(x_{1} f_{2}-x_{2} f_{1}\right)\right\}
\end{aligned}
$$

be a split-quaternion-valued function. Let $\Omega_{J}:=\Omega \cup L_{J}$, where $L_{J}=\mathbb{R}+J \mathbb{R}$. For all $p \in \Omega_{J}$, let $f_{J}: \Omega_{J} \rightarrow \mathbb{S}$ be a function

$$
f_{J}(p)=f(x+J y)=u(x, y)+J v(x, y)
$$

where $u$ and $v$ are real-valued functions. The function $f_{J}$ is called a restriction of $f$ in $\mathbb{S}$.

Remark 2.1 For any split-quaternion $p \in \mathbb{S}$, the identity function maps one split-quaternion onto itself, and there are unique $x, y \in \mathbb{R}$ with $y>0$, and $J \in \mathcal{T}$ such that $p=x+J y$, where

$$
\begin{aligned}
& x=x_{0}, \quad y=\sqrt{-x_{1}^{2}+x_{2}^{2}+x_{3}^{2}}, \\
& J=\frac{i x_{1}+j x_{2}+k x_{3}}{\sqrt{-x_{1}^{2}+x_{2}^{2}+x_{3}^{2}}}\left(x_{1}^{2}<x_{2}^{2}+x_{3}^{2}\right) .
\end{aligned}
$$

\section{Regularity of split-quaternionic functions}

Consider differential operators given by

$$
D_{l}^{*}:=\frac{\partial}{\partial x_{0}}+i \frac{\partial}{\partial x_{1}}-j \frac{\partial}{\partial x_{2}}-k \frac{\partial}{\partial x_{3}}
$$

and

$$
D_{r}^{*}:=\frac{\partial}{\partial x_{0}}+\frac{\partial}{\partial x_{1}} i-\frac{\partial}{\partial x_{2}} j-\frac{\partial}{\partial x_{3}} k,
$$


where the units $i, j$ and $k$ act from the left or right in each case. We call (3.1) the leftdifferential operator and (3.2) the right-differential operator in $\mathbb{S}$. Since both operators have similar roles, we will describe $D_{l}^{*}$ only later.

Definition 3.1 Let $\Omega$ be an open set in $\mathbb{S}$. A function $f: \Omega \rightarrow \mathbb{S}$ is said to be J-regular iffor every $J \in \mathcal{T}$, its restriction $f_{J}: \Omega_{J} \rightarrow \mathbb{S}$ is continuously differentiable and satisfies

$$
D_{J}^{*} f(x+J y)=0,
$$

where

$$
D_{J}^{*}:=\frac{1}{2}\left(\frac{\partial}{\partial x}-J \frac{\partial}{\partial y}\right)
$$

Remark 3.2 The equation $D_{J}^{*} f(x+J y)=0$ is equivalent to the following equations:

$$
\left\{\begin{array}{l}
\frac{\partial u}{\partial x}-\frac{\partial v}{\partial y}=0 \\
\frac{\partial v}{\partial x}-\frac{\partial u}{\partial y}=0 .
\end{array}\right.
$$

So, a function $f$ is $J$-regular if and only if $f$ satisfies equation (3.3).

Example 1 For $p \in \mathbb{S}$ and $a_{n} \in \mathbb{S}$ ( $n \in \mathbb{N}$, where $\mathbb{N}$ is the set of positive integers), a polyno$\operatorname{mial} f(p)=p^{n} a_{n}$ satisfies

$$
D_{J}^{*} f(p)=\frac{1}{2}\left(\frac{\partial}{\partial x}-J \frac{\partial}{\partial y}\right)(x+J y)^{n} a_{n}=0 .
$$

Hence, the polynomial $p^{n} a_{n}$ is $J$-regular.

Polynomials are used in a wide variety of fields of mathematics and every branch of chemistry and physics. In advanced mathematics, polynomials are used to construct polynomial rings and central concepts in algebra and algebraic geometry. Moreover, if a function is a solution of the equations consisting of differential operators in some systems, the function can be locally expanded as a power series by Taylor's theorem.

Proposition 3.3 The sum and product of two J-regular functions are J-regular.

Proof Let $f=u_{1}(x, y)+J \nu_{1}(x, y)$ and $g=u_{2}(x, y)+J \nu_{2}(x, y)$ be $J$-regular. Then $D_{J}^{*} f=D_{J}^{*} g=0$. From the rules of addition and product for $p=x+J y \in \mathbb{S}$, we get

$$
\begin{aligned}
D_{J}^{*}(f \pm g)= & \frac{1}{2}\left(\frac{\partial}{\partial x}-J \frac{\partial}{\partial y}\right)\left\{\left(u_{1} \pm u_{2}\right)+J\left(v_{1} \pm v_{2}\right)\right\} \\
= & \frac{1}{2}\left\{\left(\frac{\partial u_{1}}{\partial x}-\frac{\partial v_{1}}{\partial y}\right) \pm\left(\frac{\partial u_{2}}{\partial x}-\frac{\partial v_{2}}{\partial y}\right)\right\} \\
& +\frac{1}{2} J\left\{\left(-\frac{\partial u_{1}}{\partial y}+\frac{\partial v_{1}}{\partial x}\right) \mp\left(\frac{\partial u_{2}}{\partial y}-\frac{\partial v_{2}}{\partial x}\right)\right\}
\end{aligned}
$$


and

$$
\begin{aligned}
D_{J}^{*}(f g)= & \frac{1}{2}\left(\frac{\partial}{\partial x}-J \frac{\partial}{\partial y}\right)\left\{\left(u_{1} u_{2}\right)+\left(v_{1} v_{2}\right)+J\left(u_{1} v_{2}+v_{1} u_{2}\right)\right\} \\
= & \frac{1}{2}\left\{\left(\frac{\partial u_{1}}{\partial x}-\frac{\partial v_{1}}{\partial y}\right) u_{2}+u_{1}\left(\frac{\partial u_{2}}{\partial x}-\frac{\partial v_{2}}{\partial y}\right)+\left(\frac{\partial v_{1}}{\partial x}-\frac{\partial u_{1}}{\partial y}\right) v_{2}\right. \\
& \left.+v_{1}\left(\frac{\partial v_{2}}{\partial x}-v_{1} \frac{\partial u_{2}}{\partial y}\right)\right\} \\
& +\frac{1}{2} J\left\{\left(-\frac{\partial u_{1}}{\partial y}+\frac{\partial v_{1}}{\partial x}\right) u_{2}-u_{1}\left(\frac{\partial u_{2}}{\partial y}-\frac{\partial v_{2}}{\partial x}\right)-\left(\frac{\partial v_{1}}{\partial y}-\frac{\partial u_{1}}{\partial x}\right) v_{2}\right. \\
& \left.-v_{1}\left(\frac{\partial v_{2}}{\partial y}-\frac{\partial u_{2}}{\partial x}\right)\right\}
\end{aligned}
$$

respectively. Since $u_{\lambda}$ and $v_{\lambda}(\lambda=1,2)$ are real-valued functions, by using equation (3.3), we obtain $D_{J}^{*}(f \pm g)=0$ and $D_{J}^{*}(f g)=0$. Therefore, the sum and product of two $J$-regular functions are $J$-regular.

Proposition 3.4 If a function is J-regular and non-zero, then its algebraic inverse is Jregular.

Proof Let $f=u(x, y)+J v(x, y)$ be $J$-regular. Then its algebraic inverse, denoted by $(f)^{-1}$, is

$$
(f)^{-1}=\frac{f^{*}}{\mathcal{N}(f)}=\frac{u-J v}{u^{2}-v^{2}} \quad\left(u^{2} \neq v^{2}\right)
$$

Since $u$ and $v$ are real-valued functions, we have

$$
\begin{aligned}
D_{J}^{*}\left((f)^{-1}\right)= & \frac{1}{2}\left(\frac{\partial}{\partial x}-J \frac{\partial}{\partial y}\right)\left\{\frac{u-J v}{u^{2}-v^{2}}\right\} \\
= & \frac{1}{2\left(u^{2}-v^{2}\right)^{2}}\left\{\left(\frac{\partial u}{\partial x}-J \frac{\partial v}{\partial x}\right)\left(u^{2}-v^{2}\right)-(u-J v)\left(2 u \frac{\partial u}{\partial x}-2 v \frac{\partial v}{\partial x}\right)\right. \\
& \left.-J\left(\frac{\partial u}{\partial y}+\frac{\partial v}{\partial y}\right)\left(u^{2}-v^{2}\right)+J(u-J v)\left(2 u \frac{\partial u}{\partial y}-2 v \frac{\partial v}{\partial y}\right)\right\} \\
= & \frac{1}{2\left(u^{2}-v^{2}\right)^{2}}\left\{(u-J v)^{2}\left(-\frac{\partial u}{\partial x}+\frac{\partial v}{\partial y}\right)+J(u-J v)^{2}\left(-\frac{\partial v}{\partial x}+\frac{\partial u}{\partial y}\right)\right\} .
\end{aligned}
$$

From equation (3.3), we obtain $D_{J}^{*}\left((f)^{-1}\right)=0$. Therefore, the function $(f)^{-1}$ is $J$-regular.

Definition 3.5 Let $\Omega$ be an open set in $\mathbb{S}$. Let a function $f: \Omega \rightarrow \mathbb{S}$ be differentiable. Then $D_{I} f$ is said to be a J-derivative off in $\mathbb{S}$ by

$$
D_{J} f(x+J y)=\frac{1}{2}\left(\frac{\partial}{\partial x}+J \frac{\partial}{\partial y}\right) f_{J}(x+J y) .
$$

\section{Hyperholomorphy in the coordinate system}

The coordinate system used by the variables $(t, r, \theta, \varphi)$ can be represented by hyperbolic coordinates

$$
J=(\cosh \theta \sinh \varphi, \sinh \theta \sinh \varphi, \cosh \varphi) .
$$


For a split-quaternion $p=t+J r$, that is, $p=t+i r \cosh \theta \sinh \varphi+j r \sinh \theta \sinh \varphi+k r \cosh \varphi \in$ $\mathbb{S}$, a function $f: \Omega \rightarrow \mathbb{S}$ is written as follows:

$$
f(p)=u(t, r, \theta, \varphi)+J v(t, r, \theta, \varphi)
$$

We let $J_{\theta}$ and $J_{\varphi}$ be the derivatives of $J$ with respect to $\theta$ and $\varphi$, respectively, such that

$$
\begin{aligned}
& J_{\theta}=i \sinh \theta \sinh \varphi+j \cosh \theta \sinh \varphi, \\
& J_{\varphi}=i \cosh \theta \cosh \varphi+j \sinh \theta \cosh \varphi+k \sinh \varphi, \\
& J_{\theta}^{-1}=i(\sinh \varphi)^{-1} \sinh \theta+j(\sinh \varphi)^{-1} \cosh \theta
\end{aligned}
$$

and

$$
J_{\varphi}^{-1}=-i \cosh \theta \cosh \varphi-j \sinh \theta \cosh \varphi-k \sinh \varphi
$$

where $J_{\theta}^{-1}$ and $J_{\varphi}^{-1}$ are algebraic inverse elements of $J_{\theta}$ and $J_{\varphi}$, respectively, that is, the equations

$$
J_{\theta} J_{\theta}^{-1}=J_{\theta}^{-1} J_{\theta}=1
$$

and

$$
J_{\varphi} J_{\varphi}^{-1}=J_{\varphi}^{-1} J_{\varphi}=1
$$

hold.

Lemma 4.1 The left-differential operator (3.1) in this coordinate system has the form

$$
D_{l}^{*}=\frac{\partial}{\partial t}-\frac{1}{2}\left(J \frac{\partial}{\partial r}+\frac{1}{r} \frac{\partial}{\partial J}\right)
$$

where

$$
\frac{\partial}{\partial J}=\left(J_{\theta}\right)^{-1} \frac{\partial}{\partial \theta}+\left(J_{\varphi}\right)^{-1} \frac{\partial}{\partial \varphi} .
$$

Proof From the representation of $J$, we have $J r=i x_{1}+j x_{2}+k x_{3}$. Then

$$
\begin{aligned}
& \frac{\partial}{\partial r}=(-J i) \frac{\partial}{\partial x_{1}}+(J j) \frac{\partial}{\partial x_{2}}+(J k) \frac{\partial}{\partial x_{3}}=(-J)\left(i \frac{\partial}{\partial x_{1}}-j \frac{\partial}{\partial x_{2}}-k \frac{\partial}{\partial x_{3}}\right), \\
& \frac{\partial}{\partial J}=(-r i) \frac{\partial}{\partial x_{1}}+(r j) \frac{\partial}{\partial x_{2}}+(r k) \frac{\partial}{\partial x_{3}}=(-r)\left(i \frac{\partial}{\partial x_{1}}-j \frac{\partial}{\partial x_{2}}-k \frac{\partial}{\partial x_{3}}\right)
\end{aligned}
$$

and

$$
\frac{\partial}{\partial \theta}=\left(J_{\theta}\right) \frac{\partial}{\partial J}, \quad \frac{\partial}{\partial \varphi}=\left(J_{\varphi}\right) \frac{\partial}{\partial J} .
$$


Thus, we obtain

$$
i \frac{\partial}{\partial x_{1}}-j \frac{\partial}{\partial x_{2}}-k \frac{\partial}{\partial x_{3}}=\frac{1}{2}\left(-J \frac{\partial}{\partial r}+\frac{1}{-r} \frac{\partial}{\partial J}\right) .
$$

This coordinate system can be used to show that a function is hyperholomorphic. Let $p=t+J r \in \mathbb{S}(t, r \in \mathbb{R})$ and $f(p)=u(t, r, \theta, \varphi)+J v(t, r, \theta, \varphi)$, where $u$ and $v$ are real-valued functions.

Definition 4.2 Let $\Omega$ be an open set. Then a function $f=u(t, r, \theta, \varphi)+J v(t, r, \theta, \varphi)$ is said to be hyperholomorphic if $u, v \in \mathcal{C}^{1}(\Omega)$ and $f$ satisfies $D_{l}^{*} f=0$.

Theorem 4.3 A function $f(p)=u(t, r, \theta, \varphi)+J v(t, r, \theta, \varphi)$ is hyperholomorphic if and only if $u$ and $v$ satisfy the following equations:

$$
\left\{\begin{array}{l}
\frac{\partial u}{\partial t}-\frac{1}{2} \frac{\partial v}{\partial r}=0 \\
\frac{\partial v}{\partial t}-\frac{1}{2} \frac{\partial u}{\partial r}=0 \\
\frac{\partial u}{\partial J}+\frac{\partial(V)}{\partial J}=0 .
\end{array}\right.
$$

More precisely,

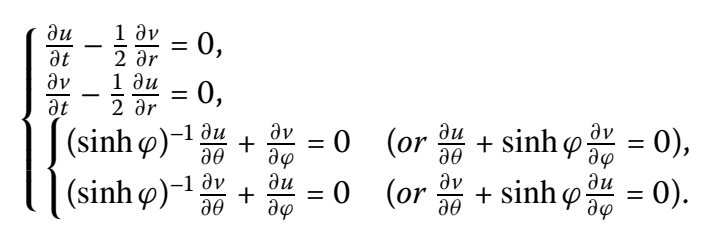

Proof From the definition of a hyperholomorphic function in split-quaternions, we have

$$
\begin{aligned}
D_{l}^{*} f & =\left(\frac{\partial}{\partial t}-\frac{1}{2} J \frac{\partial}{\partial r}-\frac{1}{2 r} \frac{\partial}{\partial J}\right)(u+J v) \\
& =\frac{\partial u}{\partial t}-\frac{1}{2} J \frac{\partial u}{\partial r}-\frac{1}{2 r} \frac{\partial u}{\partial J}+J \frac{\partial v}{\partial t}-\frac{1}{2} \frac{\partial v}{\partial r}-\frac{1}{2 r} \frac{\partial(J v)}{\partial J} \\
& =\left(\frac{\partial u}{\partial t}-\frac{1}{2} \frac{\partial v}{\partial r}\right)+J\left(\frac{\partial v}{\partial t}-\frac{1}{2} \frac{\partial u}{\partial r}\right)-\frac{1}{2 r}\left(\frac{\partial u}{\partial J}+\frac{\partial(J v)}{\partial J}\right) .
\end{aligned}
$$

Since $D_{l}^{*} f=0$, we obtain equation (4.1). Specially, the equation

$$
\frac{\partial u}{\partial J}+\frac{\partial(J v)}{\partial J}=0
$$

can be more specifically written as follows.

Since we have

$$
\begin{aligned}
\left(J_{\theta}\right)^{-1} J= & (\sinh \varphi)^{-1}(i \sinh \theta+j \cosh \theta) \\
& \times(i \cosh \theta \sinh \varphi+j \sinh \theta \sinh \varphi+k \cosh \varphi) \\
= & (\sinh \varphi)^{-1}(-i \cosh \theta \cosh \varphi-j \sinh \theta \cosh \varphi-k \sinh \varphi) \\
= & (\sinh \varphi)^{-1}\left(J_{\varphi}\right)^{-1}
\end{aligned}
$$


and

$$
\begin{aligned}
\left(J_{\varphi}\right)^{-1} J= & (-i \cosh \theta \cosh \varphi-j \sinh \theta \cosh \varphi-k \sinh \varphi) \\
& \times(i \cosh \theta \sinh \varphi+j \sinh \theta \sinh \varphi+k \cosh \varphi) \\
= & \left(\cosh ^{2} \varphi-\sinh ^{2} \varphi\right)(i \sinh \theta+j \cosh \theta) \\
= & \sinh \varphi\left(J_{\theta}\right)^{-1},
\end{aligned}
$$

we get

$$
\begin{aligned}
\frac{\partial u}{\partial J}+\frac{\partial(J v)}{\partial J}= & \left(J_{\theta}\right)^{-1} \frac{\partial u}{\partial \theta}+\left(J_{\varphi}\right)^{-1} \frac{\partial u}{\partial \varphi}+\left(J_{\theta}\right)^{-1} J \frac{\partial v}{\partial \theta}+\left(J_{\varphi}\right)^{-1} J \frac{\partial v}{\partial \varphi} \\
= & (\sinh \varphi)^{-1}\left(J_{\varphi}\right)^{-1} J \frac{\partial u}{\partial \theta}+\left(J_{\varphi}\right)^{-1} \frac{\partial u}{\partial \varphi} \\
& +(\sinh \varphi)^{-1}\left(J_{\varphi}\right)^{-1} \frac{\partial v}{\partial \theta}+\left(J_{\varphi}\right)^{-1} J \frac{\partial v}{\partial \varphi} \\
= & \left(J_{\varphi}\right)^{-1} J\left((\sinh \varphi)^{-1} \frac{\partial u}{\partial \theta}+\frac{\partial v}{\partial \varphi}\right) \\
& +\left(J_{\varphi}\right)^{-1}\left(\frac{\partial u}{\partial \varphi}+(\sinh \varphi)^{-1} \frac{\partial v}{\partial \theta}\right) \\
= & 0 .
\end{aligned}
$$

Thus, we obtain

$$
\left\{\begin{array}{l}
(\sinh \varphi)^{-1} \frac{\partial u}{\partial \theta}+\frac{\partial v}{\partial \varphi}=0 \\
(\sinh \varphi)^{-1} \frac{\partial v}{\partial \theta}+\frac{\partial u}{\partial \varphi}=0 .
\end{array}\right.
$$

Proposition 4.4 The sum and product of two hyperholomorphic functions are hyperholomorphic.

Proof Let $f=u_{1}(t, r, \theta, \varphi)+J v_{1}(t, r, \theta, \varphi)$ and $g=u_{2}(t, r, \theta, \varphi)+J(t, r, \theta, \varphi)$ be hyperholomorphic. Then $D_{l}^{*} f=D_{l}^{*} g=0$. From the rules of addition and product for $p=t+J r \in \mathbb{S}$ with the coordinate system, we get

$$
\begin{aligned}
D_{l}^{*}(f \pm g) & =\left(\frac{\partial}{\partial t}-\frac{1}{2} J \frac{\partial}{\partial r}-\frac{1}{2 r} \frac{\partial}{\partial J}\right)\left\{\left(u_{1} \pm u_{2}\right)+J\left(v_{1} \pm v_{2}\right)\right\} \\
& =\left(\frac{\partial}{\partial t}-\frac{1}{2} J \frac{\partial}{\partial r}\right)\left\{\left(u_{1} \pm u_{2}\right)+J\left(v_{1} \pm v_{2}\right)\right\}-\frac{1}{r} \frac{\partial}{\partial J}\left\{\left(u_{1} \pm u_{2}\right)+J\left(v_{1} \pm v_{2}\right)\right\} \\
& =-\frac{1}{2 r}\left(\frac{\partial u_{1}}{\partial J} \pm \frac{\partial u_{2}}{\partial J}+\frac{\partial\left(J v_{1}\right)}{\partial J} \pm \frac{\partial\left(J v_{2}\right)}{\partial J}\right)
\end{aligned}
$$

and

$$
\begin{aligned}
D_{l}^{*}(f g) & =\left(\frac{\partial}{\partial t}-\frac{1}{2} J \frac{\partial}{\partial r}-\frac{1}{2 r} \frac{\partial}{\partial J}\right)\left\{\left(u_{1} u_{2}\right)+\left(v_{1} v_{2}\right)+J\left(u_{1} v_{2}+v_{1} u_{2}\right)\right\} \\
& =\left(\frac{\partial}{\partial t}-\frac{1}{2} J \frac{\partial}{\partial r}\right)\left\{\left(u_{1} u_{2}\right)+\left(v_{1} v_{2}\right)+J\left(u_{1} v_{2}+v_{1} u_{2}\right)\right\}
\end{aligned}
$$




$$
\begin{aligned}
& -\frac{1}{2 r} \frac{\partial}{\partial J}\left\{\left(u_{1} u_{2}\right)+\left(v_{1} v_{2}\right)+J\left(u_{1} v_{2}+v_{1} u_{2}\right)\right\} \\
= & -\frac{1}{2 r}\left(\left(J_{\theta}\right)^{-1} \frac{\partial}{\partial \theta}\left\{\left(u_{1} u_{2}\right)+\left(v_{1} v_{2}\right)\right\}+\left(J_{\varphi}\right)^{-1} \frac{\partial}{\partial \varphi}\left\{\left(u_{1} u_{2}\right)+\left(v_{1} v_{2}\right)\right\}\right. \\
& +(\sinh \varphi)^{-1}\left(J_{\varphi}\right)^{-1} \frac{\partial}{\partial \theta}\left\{\left(u_{1} v_{2}\right)+\left(v_{1} u_{2}\right)\right\} \\
& \left.+(\sinh \varphi)\left(J_{\theta}\right)^{-1} \frac{\partial}{\partial \varphi}\left\{\left(u_{1} v_{2}\right)+\left(v_{1} u_{2}\right)\right\}\right) \\
= & -\frac{1}{2 r}\left(J_{\theta}\right)^{-1}\left\{u_{2}\left(\frac{\partial u_{1}}{\partial \theta}+\sinh \varphi \frac{\partial v_{1}}{\partial \varphi}\right)+u_{1}\left(\frac{\partial u_{2}}{\partial \theta}+\sinh \varphi \frac{\partial v_{2}}{\partial \varphi}\right)\right. \\
& \left.+v_{2}\left(\frac{\partial v_{1}}{\partial \theta}+\sinh \varphi \frac{\partial u_{1}}{\partial \varphi}\right)+v_{1}\left(\frac{\partial v_{2}}{\partial \theta}+\sinh \varphi \frac{\partial u_{2}}{\partial \varphi}\right)\right\} \\
& -\frac{1}{2 r}\left(J_{\varphi}\right)^{-1}\left\{u_{2}\left(\frac{\partial u_{1}}{\partial \varphi}+(\sinh \varphi)^{-1} \frac{\partial v_{1}}{\partial \theta}\right)\right. \\
& +u_{1}\left(\frac{\partial u_{2}}{\partial \varphi}+(\sinh \varphi)^{-1} \frac{\partial v_{2}}{\partial \theta}\right)+v_{2}\left(\frac{\partial v_{1}}{\partial \varphi}+(\sinh \varphi)^{-1} \frac{\partial u_{1}}{\partial \theta}\right) \\
& \left.+v_{1}\left(\frac{\partial v_{2}}{\partial \varphi}+(\sinh \varphi)^{-1} \frac{\partial u_{2}}{\partial \theta}\right)\right\}
\end{aligned}
$$

respectively. Since $u_{\lambda}$ and $v_{\lambda}(\lambda=1,2)$ are real-valued functions, by using equation (4.1), we obtain $D_{l}^{*}(f \pm g)=0$ and $D_{l}^{*}(f g)=0$. Therefore, the sum and product of two hyperholomorphic functions are hyperholomorphic.

Proposition 4.5 If a function is hyperholomorphic and non-zero, then its algebraic inverse is hyperholomorphic.

Proof Let $f=u(t, r, \theta, \varphi)+J v(t, r, \theta, \varphi)$ be hyperholomorphic. Then its algebraic inverse

$$
(f)^{-1}=\frac{f^{*}}{\mathcal{N}(f)}=\frac{u-J v}{u^{2}-v^{2}} \quad\left(u^{2} \neq v^{2}\right),
$$

where $u$ and $v$ are real-valued functions, satisfies

$$
\begin{aligned}
D_{l}^{*}\left((f)^{-1}\right)= & \left(\frac{\partial}{\partial t}-\frac{1}{2} J \frac{\partial}{\partial r}-\frac{1}{2 r} \frac{\partial}{\partial J}\right) \frac{u-J v}{u^{2}-v^{2}} \\
= & \left(\frac{\partial}{\partial t}-\frac{1}{2} J \frac{\partial}{\partial r}\right) \frac{u-J v}{u^{2}-v^{2}}-\frac{1}{2 r} \frac{\partial}{\partial J} \frac{u-J v}{u^{2}-v^{2}} \\
= & -\frac{1}{2 r}\left\{\frac{\partial}{\partial J}\left(\frac{u}{u^{2}-v^{2}}\right)-\frac{\partial}{\partial J}\left(\frac{J v}{u^{2}-v^{2}}\right)\right\} \\
= & -\frac{1}{2 r}\left(J_{\theta}\right)^{-1}\left\{\frac{\partial u}{\partial \theta}\left(u^{2}-v^{2}\right)-2 u^{2} \frac{\partial u}{\partial \theta}+2 u v \frac{\partial v}{\partial \theta}-\sinh \varphi \frac{\partial v}{\partial \varphi} u^{2}\right. \\
& \left.-\sinh \varphi \frac{\partial v}{\partial \varphi} v^{2}+\sinh \varphi \frac{\partial u}{\partial \varphi} 2 u v\right\} \frac{1}{\left(u^{2}-v^{2}\right)^{2}} \\
& -\frac{1}{2 r}\left(J_{\varphi}\right)^{-1}\left\{\frac{\partial u}{\partial \varphi}\left(u^{2}-v^{2}\right)-2 u^{2} \frac{\partial u}{\partial \varphi}+2 u v \frac{\partial v}{\partial \varphi}-(\sinh \varphi)^{-1} \frac{\partial v}{\partial \theta} u^{2}\right. \\
& \left.-(\sinh \varphi)^{-1} \frac{\partial v}{\partial \theta} v^{2}+(\sinh \varphi)^{-1} \frac{\partial u}{\partial \theta} 2 u v\right\} \frac{1}{\left(u^{2}-v^{2}\right)^{2}}
\end{aligned}
$$




$$
\begin{aligned}
= & -\frac{1}{2 r}\left(J_{\theta}\right)^{-1}\left\{\left(-u^{2}\right)\left(\frac{\partial u}{\partial \theta}+(\sinh \varphi) \frac{\partial v}{\partial \varphi}\right)\right. \\
& \left.+\left(-v^{2}\right)\left(\frac{\partial u}{\partial \theta}+(\sinh \varphi) \frac{\partial v}{\partial \varphi}\right)+(2 u v)\left(\frac{\partial v}{\partial \theta}+(\sinh \varphi) \frac{\partial u}{\partial \varphi}\right)\right\} \\
& -\frac{1}{2 r}\left(J_{\varphi}\right)^{-1}\left\{\left(-u^{2}\right)\left(\frac{\partial u}{\partial \varphi}+(\sinh \varphi)^{-1} \frac{\partial v}{\partial \theta}\right)\right. \\
& +\left(-v^{2}\right)\left(\frac{\partial u}{\partial \varphi}+(\sinh \varphi)^{-1} \frac{\partial v}{\partial \theta}\right) \\
& \left.+(2 u v)\left(\frac{\partial v}{\partial \varphi}+(\sinh \varphi)^{-1} \frac{\partial u}{\partial \theta}\right)\right\} \frac{1}{\left(u^{2}-v^{2}\right)^{2}} .
\end{aligned}
$$

From equation (4.1), we obtain $D_{l}^{*}\left((f)^{-1}\right)=0$. Therefore, the function $(f)^{-1}$ is hyperholomorphic.

Lemma 4.6 Let $\Omega$ be an open set in $\mathbb{S}$. For $p \in \Omega$, if a split-quaternionic function $f(x+J y)=$ $u(x, y)+J v(x, y)$ is hyperholomorphic, then $f$ satisfies

$$
D_{l}^{*} f=\frac{-v}{r} .
$$

Proof Since $u(x, y)$ and $v(x, y)$ are functions with respect to $x$ and $y$, the calculation of $D_{l}^{*} f$ is

$$
\begin{aligned}
D_{l}^{*} f & =\left(\frac{\partial f}{\partial t}-\frac{1}{2} J \frac{\partial f}{\partial r}\right)-\frac{1}{2 r} \frac{\partial u}{\partial J}-\frac{1}{2 r} \frac{\partial(J v)}{\partial J} \\
& =-\frac{1}{2 r}\left(\left(J_{\theta}\right)^{-1} \frac{\partial(J)}{\partial \theta} v+\left(J_{\varphi}\right)^{-1} \frac{\partial(J)}{\partial \varphi} v\right) \\
& =-\frac{v}{r} .
\end{aligned}
$$

Therefore, $f$ satisfies equation (4.2).

Theorem 4.7 For $p \in \Omega_{J}$, if a split-quaternionic function $f(x+J y)=u(x, y)+J v(x, y)$ is hyperholomorphic, then $f$ satisfies

$$
D_{l}^{*}\left(\frac{f}{r^{n}}\right)=\frac{1}{r^{n}} D_{l}^{*} f-n J \frac{f}{r^{n+1}} .
$$

Proof Since $u(x, y)$ and $v(x, y)$ are functions with respect to $x$ and $y$, the calculation of $D_{l}^{*}\left(\frac{f}{r^{n}}\right)$ is

$$
\begin{aligned}
D_{l}^{*}\left(\frac{f}{r^{n}}\right) & =\left\{\frac{\partial}{\partial t}\left(\frac{f}{r^{n}}\right)-\frac{1}{2} J \frac{\partial}{\partial r}\left(\frac{f}{r^{n}}\right)\right\}-\frac{1}{2 r} \frac{\partial}{\partial J}\left(\frac{f}{r^{n}}\right) \\
& =\frac{\partial}{\partial t}\left(\frac{f}{r^{n}}\right)-\frac{1}{2} J \frac{1}{r^{n+1}}\left\{\frac{\partial u}{\partial r} r-u n+J\left(\frac{\partial v}{\partial r} r-v n\right)\right\}-\frac{1}{r^{n+1}} v \\
& =\frac{1}{r^{n}}\left(\frac{\partial u}{\partial t}-\frac{1}{2} \frac{\partial v}{\partial r}\right)+J \frac{1}{r^{n}}\left(\frac{\partial v}{\partial t}-\frac{1}{2} \frac{\partial u}{\partial r}\right)+\frac{n}{2} J \frac{u+J v}{r^{n+1}}-\frac{1}{r^{n+1}} v \\
& =\frac{n}{2} J \frac{f}{r^{n+1}}+\frac{1}{r^{n}} D_{l}^{*} f .
\end{aligned}
$$

Therefore, we obtain the result. 
By referring to $[14,18]$ and observing Figures 1-4, we consider the corresponding Gauss theorem in four dimensions for the components of $f$ in $\mathbb{S}$. Specially, if the electric field is known, the Gauss theorem can be of help to find the division of electric charge which is inferred by integrating the electric field. For some symmetry, like cylindrical symmetry, planar symmetry, and spherical symmetry, the electric field passes through the surfaces. As shown in Figure 4, the Gauss theorem can be defined in the class containing the splitquaternionic variables with the cylindrical symmetry as a constituent. From Lemma 4.6 and Theorem 4.7, the Gauss theorem is described as follows.

Theorem 4.8 Let $f=u(x, y)+J(x, y)$ be a hyperholomorphic function, and let $K$ be any smooth and simple closed hypersurface on $\mathcal{T}$ in $\mathbb{S}$, disjoint from the real axis, $K^{*}$ being the interior of $K$. Let $n(p)=n_{0}+n_{1} i+n_{2} j+n_{3} k$, where $\left(n_{0}, n_{1}, n_{2}, n_{3}\right)$ is the unit outer normal to $K$ at $p$. Then

$$
\int_{K} n(p) f(p) \frac{1}{r^{n}} d S_{K}=-\frac{n}{2} \int_{K^{*}} J \frac{u}{r^{n+1}} d V
$$

where $d S_{k}$ is the element of surface area on $K$.

Proof Suppose that $f$ is hyperholomorphic and $n(p)=n_{0}+n_{1} i+n_{2} j+n_{3} k$, where $\left(n_{0}, n_{1}, n_{2}, n_{3}\right)$ is the unit outer normal to $K$ at $p$ (such as Figure 4). From Theorem 4.7, we have

$$
\begin{aligned}
\int_{K} n(p) f(p) \frac{1}{r^{n}} d S_{K} & =\int_{K} \frac{1}{r^{n}}\left(n_{0} f+i n_{1} f+j n_{2} f+k n_{3} f\right) d S_{K} \\
& =\int_{K^{*}} D_{l}\left(\frac{f}{r^{n}}\right) d V=\int_{K^{*}}\left(\frac{1}{r^{n}} D_{l} f-\frac{n}{2} J \frac{f}{r^{n+1}}\right) d V \\
& =\int_{K^{*}}\left(-\frac{n}{2} J \frac{u}{r^{n+1}}+\frac{-n}{2 r^{n}} \frac{-v}{r}\right) d V \\
& =\int_{K^{*}}\left(-\frac{n}{2} J \frac{u}{r^{n+1}}\right) d V .
\end{aligned}
$$

Thus, we can obtain the result.

Competing interests

The author declares that they have no competing interests.

\section{Acknowledgements}

This work was supported by the Dongguk University Research Fund of 2017.

\section{Publisher's Note}

Springer Nature remains neutral with regard to jurisdictional claims in published maps and institutional affiliations.

Received: 13 November 2016 Accepted: 6 March 2017 Published online: 17 March 2017

\section{References}

1. Hamilton, WR: Elements of Quaternions. Longmans, Green, and Company, London (1899)

2. Clifford, WK: Preliminary sketch of bi-quaternions. J. Lond. Math. Soc. 4, 381-395 (1873)

3. Fueter, R: Die Funktionentheorie der Differentialgleichungen $\Delta u=0$ und $\Delta \Delta u=0$ mit vier reellen Variablen. Comment. Math. Helv. 7(1), 307-330 (1934)

4. Fueter, R: Über die analytische Darstellung der regulären Funktionen einer Quaternionenvariablen. Comment. Math. Helv. 8(1), 371-378 (1935) 
5. Kim, JE, Lim, SJ, Shon, KH: Regular functions with values in ternary number system on the complex Clifford analysis. Abstr. Appl. Anal. 2013, Article ID 136120 (2013)

6. Kim, JE, Lim, SJ, Shon, KH: Regularity of functions on the reduced quaternion field in Clifford analysis. Abstr. Appl. Anal. 2014, Article ID 654798 (2014)

7. Cockle, J: LII. On systems of algebra involving more than one imaginary; and on equations of the fifth degree. Lond. Edin. Dub. Phil. Mag. J. Sci. 35(238), 434-437 (1849)

8. Karzel, H, Kist, G: Kinematic Algebras and Their Geometries, Rings and Geometry, pp. 437-509. Springer, Dordrecht (1985)

9. Frenkel, I, Libine, M: Quaternionic analysis, representation theory and physics. Adv. Math. 218(6), 1806-1877 (2008)

10. Libine, M: An invitation to split quaternionic analysis. In: Hypercomplex Analysis and Applications, pp. 161-180. Springer, Basel (2011)

11. Kim, JE, Shon, KH: Polar coordinate expression of hyperholomorphic functions on split quaternions in Clifford analysis. Adv. Appl. Clifford Algebras 25(4), 915-924 (2015)

12. Kim, JE, Shon, KH: The regularity of functions on dual split quaternions in Clifford analysis. Abstr. Appl. Anal. 2014, Article ID 369430 (2014)

13. Kim, JE, Shon, KH: Inverse mapping theory on split quaternions in Clifford analysis. Filomat 30(7), 1883-1890 (2016)

14. Cullen, CG: An integral theorem for analytic intrinsic functions on quaternions. Duke Math. J. 32(1), 139-148 (1965)

15. Leutwiler, H: Modified quaternionic analysis in $\mathbb{R}^{3}$. Complex Var. Elliptic Equ. 20(1-4), 19-51 (1992)

16. Gentili, G, Struppa, DC: A new theory of regular functions of a quaternionic variable. Adv. Math. 216(1), 279-301 (2007)

17. Alayón-Solarz, D: Cullen-regular quaternionic functions in a Fueter operator framework (2008), arXiv:0805.0141v2 [math.CV]

18. Alayón-Solarz, D: A generalization of a Cullen's integral theorem for the quaternions. Bol. Asoc. Mat. Venez. 16(1), 5-9 (2009)

19. Marin, M, Marinescu, C: Thermoelasticity of initially stressed bodies, asymptotic equipartition of energies. Int. J. Eng Sci. 36(1), 73-86 (1998)

20. Marin, M, Lupu, M: On harmonic vibrations in thermoelasticity of micropolar bodies. J. Vib. Control 4(5), 507-518 (1998)

21. Marin, M: A domain of influence theorem for microstretch elastic materials. Nonlinear Anal., Real World Appl. 11(5), 3446-3452 (2010)

\section{Submit your manuscript to a SpringerOpen ${ }^{\circ}$ journal and benefit from:}

- Convenient online submission

- Rigorous peer review

- Immediate publication on acceptance

Open access: articles freely available online

- High visibility within the field

- Retaining the copyright to your article 